\title{
Term algebras of elementarily equivalent atom structures
}

\author{
Andréka, H. and Németi, I.
}

We dedicate this paper to Bjarni Jónsson.

\begin{abstract}
We exhibit two relation algebra atom structures such that they are elementarily equivalent but their term algebras are not. This answers Problem 14.19 in the book Hirsch, R. and Hodkinson, I., "Relation Algebras by Games", North-Holland, 2002.
\end{abstract}

\section{Introduction}

Atom structures for Boolean algebras with operators, and in particular for relation algebras, were introduced in Jónsson-Tarski [7]. These structures proved to be a central tool in algebraic logic, see, e.g., [2], 3, section 19], 4, section 2.7], [5, section 2.7], 8, 9, 10]. There is a kind of duality between atom structures of algebras and complex algebras of relational structures, a large part of this duality is elaborated in [7]. Atom structures are useful in constructing relation algebras, because atom structures are simpler and hence easier to work with. Therefore, it is useful to see what properties of complex algebras can be ensured by constructing appropriate atom structures. Representability of the complex algebra is not such a property, because there are two elementarily equivalent relation algebra atom structures such that the complex algebra of one is representable while that of the other is not, a result of Hirsch and Hodkinson [5, Corollary 14.14].

The term algebra of an atom structure is the smallest subalgebra of its complex algebra that has the same atom structure: it is the subalgebra of the complex algebra generated by the singletons of the atoms. They are more tightly connected to their atom structures, e.g., representability of the 
term algebra of a relation algebra atom structure can be ensured by its firstorder logic theory. So, if two atom structures are elementarily equivalent, then their term algebras are either both representable, or neither of them is representable (a result of Venema [11], see [5, Theorem 2.84]). If two atom structures are "very close", i.e., if they are $L_{\infty, \omega}$-indistinguishable, then their term algebras are also this very close, i.e., they are $L_{\infty, \omega}$-indistinguishable ([5, Exercise 14.6]). Problem 14.19 in [5] asks if this last result holds with $L_{\omega \omega}$ in place of $L_{\infty, \omega}$ : If $S, S^{\prime}$ are elementarily equivalent relation algebra atom structures, must the term algebras of $S$ and $S^{\prime}$ also be elementarily equivalent?

In this paper we give a negative answer to this question. We construct a relation algebra atom structure $S$, such that the term algebra of $S$ and that of an ultraproduct of $S$ are not elementarily equivalent. Moreover, $S$ is an atom structure of a completely representable simple relation set algebra.

\section{The construction}

We begin by recalling terminology from [5]. A (relational algebra type) atom structure is a structure $\langle S, P, C, I\rangle$, where $P, C$ and $I$ are ternary, binary, and unary relations on $S$, respectively. The complex algebra of an atom structure $\langle S, P, C, I\rangle$ is the algebra $\left\langle A,+,-, ;,{ }^{\smile}, 1^{\prime}\right\rangle$ where $A$ is the collection of all subsets of $S,+$ and - are the operations of forming union and complement (with respect to $S$ ) respectively, and the operations ; , $`, 1^{\prime}$ are determined by $P, C, I$ as follows. Let $X, Y \subseteq S$. Then $X ; Y=\{u: P(x, y, u)$ for some $x \in$ $X, y \in Y\}, X^{\smile}=\{u: C(x, u)$ for some $x \in X\}$, and $1^{\prime}=I$. The atom structure is called completely representable if its complex algebra is completely representable, that is to say if, up to an isomorphism, $A$ is a set of binary relations such that the biggest element is the union (as opposed to the supremum only) of the atomic relations, and the operations,,$+- ;,,^{\prime}, 1^{\prime}$ are the following standard operations on binary relations: union, complement (with respect to a largest element of $A$ ), relation composition, converse, and the identity relation. Finally, the term algebra of an atom structure is the subalgebra of its complex algebra generated by the singletons. In the paper, $\omega$ denotes the set of non-negative integers.

Theorem 1 There are completely representable relational algebra atom structures which are elementarily equivalent but their term algebras are not elementarily equivalent. 
Proof. First we define a relation algebra type atom structure $\mathcal{S}=\langle S, P, C, I\rangle$. The universe $S$ of the atom structure is

$$
\begin{aligned}
& \left\{\operatorname{Id}_{n, i}: n \in \omega, i \leq n\right\} \cup \\
& \left\{r_{n, k}: n \in \omega, 1 \leq k \leq n\right\} \cup\left\{r_{n, k}^{-}: n \in \omega, 1 \leq k \leq n\right\} \cup \\
& \left\{w_{n, i, m, j}: n, m \in \omega, i \leq n, j \leq m\right\},
\end{aligned}
$$

and $I=\left\{\operatorname{ld}_{n, i}: n \in \omega, i \leq n\right\}$. The binary relation $C$ is

$$
\begin{aligned}
& \{(x, x): x \in I\} \cup \\
& \left\{\left(r_{n, k}, r_{n, k}^{-}\right): n \in \omega, 1 \leq k \leq n\right\} \cup\left\{\left(r_{n, k}^{-}, r_{n, k}\right): n \in \omega, 1 \leq k \leq n\right\} \cup \\
& \left\{\left(w_{n, i, m, j}, w_{m, j, n, i}\right): n, m \in \omega, i \leq n, j \leq m\right\} .
\end{aligned}
$$

To define $P$, we first define two unary operations on $S$, the domain $\operatorname{dm}($ a) and the range $\operatorname{rg}(\mathrm{a})$ of $a$ :

$$
\begin{aligned}
& \operatorname{dm}(\mathrm{x})=\operatorname{rg}(\mathrm{x})=x \text { for each } x \in I, \\
& \operatorname{dm}\left(\mathrm{r}_{\mathrm{n}, \mathrm{k}}\right)=\operatorname{rg}\left(\mathrm{r}_{\mathrm{n}, \mathrm{k}}^{-}\right)=\mathrm{Id}_{n, 0}, \quad \operatorname{rg}\left(\mathrm{r}_{\mathrm{n}, \mathrm{k}}\right)=\operatorname{dm}\left(\mathrm{r}_{\mathrm{n}, \mathrm{k}}^{-}\right)=\mathrm{Id}_{n, k}, \quad \text { and } \\
& \operatorname{dm}\left(\mathrm{w}_{\mathrm{n}, \mathrm{i}, \mathrm{m}, \mathrm{j}}\right)=\operatorname{rg}\left(\mathrm{w}_{\mathrm{m}, \mathrm{j}, \mathrm{n}, \mathrm{i}}\right)=\mathrm{Id}_{n, i} .
\end{aligned}
$$

Now, the ternary relation $P$ on $S$ is:

$$
\begin{aligned}
& \{(\operatorname{dm}(\mathrm{a}), a, a): a \in S\} \cup\{(a, \operatorname{rg}(\mathrm{a}), a): a \in S\} \cup\{(a, b, \operatorname{dm}(\mathrm{a})):(a, b) \in C\} \cup \\
& \{(a, b, c): a, b, c \in S \backslash I, \operatorname{dm}(\mathrm{a})=\operatorname{dm}(\mathrm{c}), \operatorname{rg}(\mathrm{a})=\operatorname{dm}(\mathrm{b}), \operatorname{rg}(\mathrm{b})=\operatorname{rg}(\mathrm{c})\} .
\end{aligned}
$$

With this, the atom structure $\mathcal{S}$ has been defined.

Here is a short intuitive description of $\mathcal{S}$, see Figure 1. Keeping the complex algebra in mind, we call the elements of $S$ atoms, the elements of $I$ identity atoms, and the rest diversity atoms, and we say that $a$ goes from $\operatorname{dm}($ a) to $\operatorname{rg}(\mathrm{a})$. Now, between any two identity atoms in $\mathcal{S}$ only one or two diversity atoms go. The pairs of identity atoms that have two diversity atoms between them (in both directions) are the pairs $\operatorname{ld}_{n, 0}$ and $\operatorname{ld}_{n, k}$ with $1 \leq k \leq n$, we call these splitable pairs and the atoms going between them are called split atoms: from $\mathrm{Id}_{n, 0}$ to $\mathrm{Id}_{n, k}$ the two diversity atoms $r_{n, k}$ and $w_{n, 0, n, k}$ go, and from $\mathbf{I d}_{n, k}$ to $\operatorname{ld}_{n, 0}$ their converses, $r_{n, k}^{-}$and $w_{n, k, n, 0}$. Between $\mathrm{Id}_{n, i}$ and $\mathrm{Id}_{n, i}$ two atoms go, the identity atom $\mathrm{Id}_{n, i}$ and the diversity atom 
$w_{n, i, n, i}$. Between $\operatorname{Id}_{n, i}$ and $\operatorname{Id}_{m, j}$ when $n \neq m$ or $0 \notin\{i, j\}$ only one atom goes, namely $w_{n, i, m, j}$.

The pair of the two diversity atoms $r_{n, k}$ and $w_{n, 0, n, k}$ going from $\operatorname{Id}_{n, 0}$ to $\mathrm{Id}_{n, k}$ is called a leaf. These leaves are connected at $\operatorname{ld}_{n, 0}$, and we call them a plant emanating from $\operatorname{ld}_{n, 0}$. So, intuitively, $\mathcal{S}$ consists of infinitely many plants, one plant with $n$ leaves for all natural number $n$, see Figure 1.
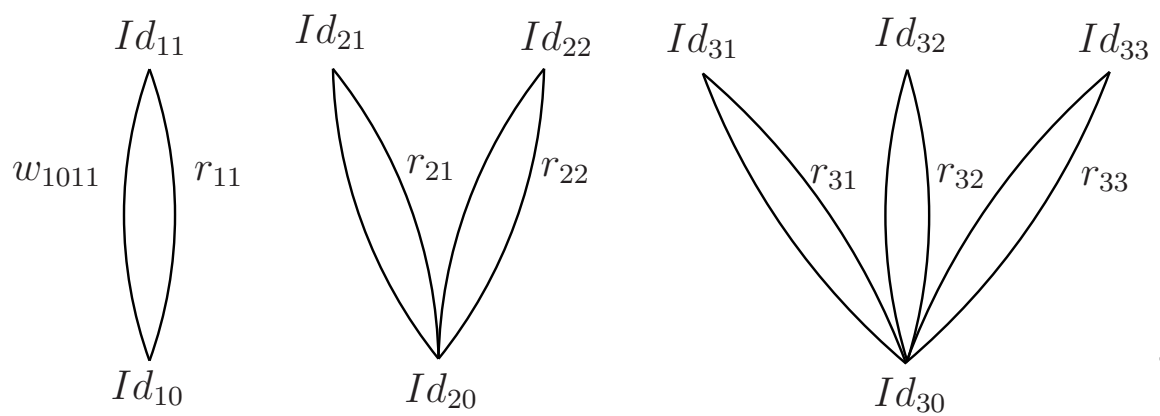

Figure 1: The split atoms in the atom structure $\mathcal{S}$.

All the atoms of $\mathcal{S}$ are "big" in the sense that their products in the complex algebra are as big as possible allowing representability. At the end of this proof we show that the complex algebra of $\mathcal{S}$ is indeed completely representable, so it is a relation algebra atom structure.

Let $\mathcal{S}^{\prime}$ be a nontrivial ultrapower of $\mathcal{S}$. Then $\mathcal{S}, \mathcal{S}^{\prime}$ are elementarily equivalent by the fundamental theorem of ultraproducts. We will show that their term algebras are not elementarily equivalent, by exhibiting a formula that distinguishes them, as follows. The ultrapower $\mathcal{S}^{\prime}$ looks exactly like $\mathcal{S}$, there are leaves grouped into plants, except that in $\mathcal{S}^{\prime}$ there are plants with infinitely many leaves, too. The key idea is that in the term algebras, the finite and infinite plants can be distinguished by a first-order logic formula. Namely, only finitely many leaves can be "split" by any element of the term algebra. Thus, for an infinite plant there is no element that splits all its leaves, while for a finite plant clearly there is such an element. Using this, we can express that in $\mathcal{S}$ all the plants are finite while in $\mathcal{S}^{\prime}$ there are infinite plants, too. This will be a first-order logic formula distinguishing $\mathcal{S}$ and $\mathcal{S}^{\prime}$. We note that in the complex algebra of $\mathcal{S}^{\prime}$ there are elements splitting all the leaves of the infinite plants, too, but these elements cannot be generated from singletons, so they are not in the term algebra. 
We now turn to elaborating the details of the above plan. Let $\mathfrak{A}, \mathfrak{A}^{\prime}$ denote the term algebras of $\mathcal{S}, \mathcal{S}^{\prime}$, respectively. Let the operations of the term algebras be denoted as follows: $\cdot,+,-, 0,1$ denote Boolean intersection, addition, complementation, zero and unit, and let ; , ${ }^{\prime}, 1^{\prime}$ denote relation algebraic composition, conversion and identity element, respectively. For $a, x, y \in A$ we say that a splits $x, y$ if $x, y$ are distinct identity atoms and there are atoms going from $x$ to $y$ both in $a$ and in its complement. Formally, let

$$
\begin{aligned}
& x \times y:=x ; 1 \cdot 1 ; y, \\
& \sigma(a, x, y): \Leftrightarrow \\
& \quad x, y \text { are distinct identity atoms and } a \cdot(x \times y) \neq 0,-a \cdot(x \times y) \neq 0 .
\end{aligned}
$$

Thus, if $a$ splits $x, y$, then $x, y$ is a splitable pair of identity atoms. Next we prove by an easy induction that each element in $A^{\prime}$ splits only finitely many pairs of atoms. Let the induction statement be denoted by $F(a)$,

$F(a): \Leftrightarrow a$ splits only finitely many pairs of identity atoms.

Since $\mathfrak{A}^{\prime}$ is a term algebra, it is generated by its atoms. When $a$ is an atom, it can split only one pair of atoms, namely it can split only the pair dm(a), rg(a) of its domain and range. Thus, $F(a)$ holds for all atoms $a$.

Assume that $F(a), F(b)$ hold. By definition, $a$ splits $x, y$ iff $-a$ splits $x, y$, so $F(-a)$ holds, too. It is easy to see that if $a+b$ splits $x, y$ then at least one of $a$ and $b$ splits $x, y$. So $a+b$ can split only those pairs of atoms that are split either by $a$ or by $b$, hence $F(a)$ and $F(b)$ imply $F(a+b)$.

$F\left(1^{\prime}\right)$ holds, because $1^{\prime}$ cannot split any pair of distinct atoms (since $\operatorname{dm}(\mathrm{a})=\operatorname{rg}(\mathrm{a})$ for identity atoms $)$.

If $a$ splits $x, y$, then $a^{\smile}$ splits $y, x$, so if $F(a)$ holds, then also $F\left(a^{\smile}\right)$ holds.

We set to show $F(a ; b)$. First notice that the product $a ; b$ of two diversity atoms splits no pair of atoms in $\mathfrak{A}$, and this fact can be expressed by a first-order logic formula about $\mathcal{S}$ :

$$
\begin{aligned}
& {[a, b \notin I \wedge x, y \in I \wedge x \neq y \wedge P(a, b, c) \wedge P(x, c, c) \wedge P(x, d, d) \wedge P(c, y, c) \wedge} \\
& P(d, y, d)] \rightarrow P(a, b, d) .
\end{aligned}
$$

Therefore this same formula holds in $\mathcal{S}^{\prime}$, too, since $\mathcal{S}^{\prime}$ is an ultrapower of $\mathcal{S}$, and so the product $a ; b$ of two diversity atoms splits no pair of atoms in $\mathfrak{A}^{\prime}$, either. This implies 


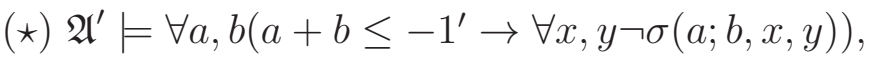

as follows. Let $x, y$ be a pair of distinct identity atoms, and let $X, Y \subseteq S^{\prime}$ be subsets of diversity atoms. Then $X ; Y=\bigcup\{a ; b: a \in X, b \in Y\}$, in $\mathfrak{A}^{\prime}$. Now, assume that $x \times y \cap X ; Y \neq 0$. Then $x \times y \cap a ; b \neq 0$ for some $a \in X, b \in Y$. Since $a, b$ are diversity atoms, they do not split $x, y$, thus $x \times y \subseteq a ; b \subseteq X ; Y$, which means that $X ; Y$ does not split $x, y$, either. This proves $(\star)$.

Let now $a, b \in A^{\prime}$ be arbitrary. Let $a^{\prime}:=a \cdot 1^{\prime}, a^{\prime \prime}:=a \cdot-1^{\prime}$, and the same for $b$, i.e., $b^{\prime}:=b \cdot 1^{\prime}, b^{\prime \prime}:=b \cdot-1^{\prime}$. Then $a ; b=a^{\prime} ; b+a ; b^{\prime}+a^{\prime \prime} ; b^{\prime \prime}$. Hence, by the previously proven case for addition, $a ; b$ can split only those pairs of atoms that are split by either one of $a^{\prime} ; b, a ; b^{\prime}$ or $a^{\prime \prime} ; b^{\prime \prime}$. Assume that $x, y$ are distinct identity atoms. By the above statement $(\star)$, then $a^{\prime \prime} ; b^{\prime \prime}$ does not split $x, y$. We show that if $a^{\prime} ; b$ splits $x, y$, then $b$ also splits them. Indeed, let $r, s$ be atoms going from $x$ to $y$ such that $r \leq a^{\prime} ; b$ and $s \leq-\left(a^{\prime} ; b\right)$. Then $r \leq b$ since $a^{\prime} ; b \leq b$ by $a^{\prime} \leq 1^{\prime}$. Also we must have $x \leq a^{\prime}$. Assume $s \leq b$. Then $s \leq x ; b \leq a^{\prime} ; b$, contradicting our assumption $s \leq-\left(a^{\prime} ; b\right)$. Similarly, if $a ; b^{\prime}$ splits $x, y$ then $a$ also splits them. Thus, $a ; b$ can only split those atoms that are split by $a$ or by $b$, hence $F(a ; b)$ holds by $F(a), F(b)$. We have proved that $F(a)$ holds for all $a \in A^{\prime}$.

We are ready to exhibit the sentence distinguishing $\mathfrak{A}$ and $\mathfrak{A}^{\prime}$. This sentence states that for all atoms $x$ there is an element $a$ that splits all pairs $x, y$ if they are splitable. Formally, let

$$
\varphi \quad: \Leftrightarrow \quad \forall x \exists a \forall y(\exists a \sigma(a, x, y) \rightarrow \sigma(a, x, y)) .
$$

Now, $\mathfrak{A} \models \varphi$ because for all $x$ there are only finitely many $y$ such that $x, y$ is splitable. On the other hand, assume that $\mathcal{S}^{\prime}$ is the ultrapower of $\mathcal{S}$ by the ultrafilter $D$ on $J$, and let

$$
x:=\left\langle\mathrm{Id}_{n, 0}: n \in J\right\rangle / D .
$$

Then $x$ is an identity atom in $\mathfrak{A}^{\prime}$ such that infinitely many "leaves" emanate from $x$. Since all elements $a$ of $\mathfrak{A}^{\prime}$ can split only finitely many of these leaves, there is no element in $\mathfrak{A}^{\prime}$ that can split all of the splitable atoms $x, y$, thus $\mathfrak{A}^{\prime} \not \forall \varphi$. We have shown that $\mathfrak{A}$ and $\mathfrak{A}^{\prime}$ are not elementarily equivalent.

Finally, we show that $\mathcal{S}$ and $\mathcal{S}^{\prime}$ are completely representable. It is enough to show that $\mathcal{S}$ is completely representable, since an ultraproduct of completely representable atom structures is again completely representable (see [5. Exercise 14.1]). 
Let $U$ be a set and $R, T \subseteq U \times U$ be binary relations on $U$. Relation composition $R \mid T$ of relations $R$ and $T$, converse $R^{-1}$ of relation $R$ and the identity relation $\operatorname{ld}_{U}$ on $U$ are defined as follows.

$$
\begin{aligned}
& R \mid T=\{(u, v): \exists w[(u, w) \in R \text { and }(w, v) \in T]\} \\
& R^{-1}=\{(v, u):(u, v) \in R\} \\
& \operatorname{ld}_{U}=\{(u, u): u \in U\} .
\end{aligned}
$$

The relation $U \times U \backslash \mathrm{Id}_{U}$ is called the diversity relation on $U$.

To show complete representability of $\mathcal{S}$, we will construct a set $U$ and a function rep : $S \rightarrow U \times U$ such that

(i) $\langle\operatorname{rep}(a): a \in S\rangle$ is a partition of $U \times U$ to nonempty parts,

(ii) $a \in I \quad$ iff $\quad \operatorname{rep}(a) \subseteq \operatorname{ld}_{U} \quad$ iff $\quad \operatorname{rep}(a) \cap \operatorname{Id}_{U} \neq \emptyset$,

(iii) $C(a, b)$ iff $\quad \operatorname{rep}(b)=\operatorname{rep}(a)^{-1} \quad$ iff $\quad \operatorname{rep}(b) \cap \operatorname{rep}(a)^{-1} \neq \emptyset$,

(iv) $P(a, b, c)$ iff $\operatorname{rep}(c) \subseteq \operatorname{rep}(a) \mid \operatorname{rep}(b)$ iff $\operatorname{rep}(c) \cap(\operatorname{rep}(a) \mid \operatorname{rep}(b)) \neq \emptyset$.

$U$ consists of countably many disjoint copies of $\omega$ : let $U_{n, i}=\omega \times\{(n, i)\}$, and

$$
U:=\bigcup\left\{U_{n, i}: n \in \omega, i \leq n\right\} .
$$

We define for $n, m \in \omega, i \leq n, j \leq m$

$$
\begin{aligned}
& \operatorname{rep}\left(\operatorname{Id}_{n, i}\right):=\left\{(u, u): u \in U_{n, i}\right\}, \quad \operatorname{rep}\left(w_{n, i, n, i}\right):=U_{n, i} \times U_{n, i} \backslash \operatorname{Id}_{n, i}, \\
& \operatorname{rep}\left(w_{n, i, m, j}\right):=U_{n, i} \times U_{m, j} \quad \text { when } n \neq m, \text { or } i \neq j \text { and } 0 \notin\{i, j\} .
\end{aligned}
$$

It remains to define $\operatorname{rep}(s)$ for the "split" atoms $s$ (i.e., for the atoms that form leaves). Assume $n, k \in \omega$ and $1 \leq k \leq n$ (and $i, j \in \omega$ are arbitrary).

$$
\begin{aligned}
& \operatorname{rep}\left(r_{n, k}\right):= \\
& \quad\left\{\left\langle(i, n, 0),\left(2^{i} 3^{j}, n, k\right)\right\rangle: i, j \in \omega\right\} \cup\left\{\left\langle(i, n, 0),\left(2^{j} 3^{i}, n, k\right)\right\rangle: i, j \in \omega\right\} \cup \\
& \quad\left\{\left\langle\left(2^{i} 3^{j}, n, 0\right),(i, n, k)\right\rangle: i, j \in \omega\right\} \cup\left\{\left\langle\left(2^{j} 3^{i}, n, 0\right),(i, n, k)\right\rangle: i, j \in \omega\right\} \cup \\
& \quad\left\{\left\langle\left(2^{i} 5^{k}, n, 0\right),(i, n, k)\right\rangle: i, j \in \omega\right\} . \\
& \operatorname{rep}\left(w_{n, 0, n, k}\right):=U_{n, 0} \times U_{n, k} \backslash \operatorname{rep}\left(r_{n, k}\right), \\
& \operatorname{rep}\left(r_{n, k}^{-}\right):=\operatorname{rep}\left(r_{n, k}\right)^{-1}, \quad \operatorname{rep}\left(w_{n, k, n, 0}\right):=\operatorname{rep}\left(w_{n, 0, n, k}\right)^{-1} .
\end{aligned}
$$


The properties of $R:=\operatorname{rep}\left(r_{n, k}\right)$ that we will use are the following. Let $T:=U_{n, 0} \times U_{n, k} \backslash R$. Assume $X, Y \in\{R, T\}$ and $1 \leq \ell \leq n, k \neq \ell$.

(r1) For each $u \in U_{n, 0}$ there are at least two $v \in U_{n, k}$ such that $(u, v) \in X$.

(r2) For each $v \in U_{n, k}$ there are at least two $u \in U_{n, 0}$ such that $(u, v) \in X$.

(r3) For all distinct $u, v \in U_{n, 0}$ there is $w \in U_{n, k}$ such that $(u, w) \in X$ and $(v, w) \in Y$.

(r4) For all distinct $u, v \in U_{n, k}$ there is $w \in U_{n, 0}$ such that $(w, u) \in X$ and $(w, v) \in Y$.

(r5) For all $u \in U_{n, k}$ and $v \in U_{n, \ell}$ there is $w \in U_{n, 0}$ such that $(w, u) \in X$ and $(w, v) \in Y$.

Now, (r1) follows from (r3) and (r2) follows from (r4). To check (r3), let $u, v \in U_{n, 0}$ be distinct. Assume $u=(i, n, 0)$ and $v=(j, n, 0)$. Then $i \neq j$ and $(u, w) \in R,(v, w) \in R$ for $w=\left(2^{i} 3^{j}, n, k\right)$ by the first line in the definition of $R$. Let $w=\left(2^{i} 3^{q}, n, k\right)$ where $q=j+1$. Then $(u, w) \in R$ by the first line in the definition of $R$. To show $(v, w) \in T$ we have to show $(v, w) \notin R$. This last statement is true because $j \notin\{i, q\}$, so the pair $(v, w)$ is not included in $R$ by the first line of its definition, and $j<2^{i} 3^{q}$ by $q=j+1$, so the pair $(v, w)$ is not included in $R$ by the second and third lines of its definition. Similarly, $(u, w) \in T,(v, w) \in R$ for $w=\left(2^{q} 3^{j}, n, k\right)$ where $q=i+1$. Finally, $(u, w) \in T,(v, w) \in T$ for $w=\left(2^{q} 3^{t}, n, k\right)$ where $q, t$ are both bigger than $i+j$. The proof for (r4) is slightly more involved than the proof of (r3) because of the last line in the definition of $R$. Let $u, v \in U_{n, k}$ be distinct, say $u=(i, n, k)$ and $v=(j, n, k)$ with $i \neq j$. Then $(w, u) \in R$ and $(w, v) \in R$ for $w=\left(2^{i} 3^{j}, n, 0\right)$ by the second line in the definition of $R$. Let $w=\left(2^{i} 3^{q}, n, k\right)$ where $q=j+1$. Then $(w, u) \in R$ by the second line in the definition of $R$. Also, the pair $(w, v)$ is not included in $R$ by the first line of its definition since $2^{i} 3^{q}>j$, it is not included in $R$ by the second line since $j \notin\{i, q\}$, and it is not included by the third line since $5^{k}$ is not a divisor of $2^{i} 3^{q}$. The rest of (r4) and the case of ( 55$)$ when $i \neq j$ are similar. The third line in the definition of $R$ is present for the case of ( $\mathrm{r} 5)$ when $i=j$ : assume $u=(i, n, k)$ and $v=(i, n, \ell)$, and let $w=\left(2^{i} 5^{k}, n, 0\right)$. Then $(w, u) \in R$ by the third line in the definition of $R$ and $(w, v) \notin R$ because $i<2^{i} 5^{k}$ and $5^{k}$ is a divisor of $2^{i} 5^{k}$ but $5^{\ell}$ is not a divisor of $2^{i} 5^{k}$. 
We are ready to check (i)-(iv). To check (i)-(iii) is straightforward. It is also straightforward to check (iv) when $a, b, c$ are all diversity atoms that do not form leaves. It remains to check the following when $k, \ell \neq 0, k \neq \ell$.

$$
\begin{aligned}
& \text { (iv.1) } \operatorname{rep}\left(w_{n, 0, n, 0}\right) \mid \operatorname{rep}(a) \supseteq \operatorname{rep}(b) \quad \text { when } a, b \in\left\{r_{n, k}, w_{n, 0, n, k}\right\}, \\
& \text { (iv.2) } \operatorname{rep}\left(w_{n, k, n, k}\right) \mid \operatorname{rep}(a) \supseteq \operatorname{rep}(b) \quad \text { when } a, b \in\left\{r_{n, k}^{-}, w_{n, k, n, 0}\right\}, \\
& \text { (iv.3) } \operatorname{rep}\left(w_{n, k, n, \ell}\right) \mid \operatorname{rep}(a) \supseteq \operatorname{rep}(b) \quad \text { when } a \in\left\{r_{n, \ell}^{-}, w_{n, \ell, n, 0}\right\} \text { and } b \in \\
& \quad\left\{r_{n, k}^{-}, w_{n, k, n, 0}\right\}, \\
& \text { (iv.4) } \operatorname{rep}(a) \mid \operatorname{rep}(b) \supseteq\left\{(u, v) \in U_{n, 0} \times U_{n, 0}: u \neq v\right\} \quad \text { when } a \in\left\{r_{n, k}, w_{n, 0, n, k}\right\} \\
& \quad \text { and } b \in\left\{r_{n, k}^{-}, w_{n, k, n, 0}\right\}, \\
& \text { (iv.5) } \operatorname{rep}(a) \mid \operatorname{rep}(b) \supseteq\left\{(u, v) \in U_{n, k} \times U_{n, k}: u \neq v\right\} \quad \text { when } a \in\left\{r_{n, k}^{-}, w_{n, k, n, 0}\right\} \\
& \quad \text { and } b \in\left\{r_{n, k}, w_{n, 0, n, k}\right\}, \\
& \text { (iv.6) } \operatorname{rep}(a) \mid \operatorname{rep}(b) \supseteq U_{n, k} \times U_{n, \ell} \quad \text { when } a \in\left\{r_{n, k}^{-}, w_{n, k, n, 0}\right\} \text { and } b \in \\
& \quad\left\{r_{n, \ell}, w_{n, 0, n, \ell}\right\} .
\end{aligned}
$$

Of the above, (iv.1) is true because of $(\mathrm{r} 2)$ and because rep $\left(w_{n, 0, n, 0}\right)$ is the diversity relation on $U_{n, 0}$ : assume $(u, v) \in \operatorname{rep}(b) \subseteq U_{n, 0} \times U_{n, k}$. There is $(w, v) \in \operatorname{rep}(a), w \neq u$ because $(\mathrm{r} 2)$. Then $w \in U_{n, 0}$ because the domain of $\operatorname{rep}(a)$ is $U_{n, 0}$ and $(w, v) \in \operatorname{rep}\left(w_{n, 0, n, 0}\right)$ because $w \neq u$ and $\operatorname{rep}\left(w_{n, 0, n, 0}\right)$ is the diversity relation on $U_{n, 0}$. Hence, $(u, v) \in\{(u, w)\}\left|\{(w, v)\} \subseteq \operatorname{rep}\left(w_{n, 0, n, 0}\right)\right|$ $\operatorname{rep}(a)$. The proofs of (iv.2) and (iv.3) are analogous. To show (iv.4), let $u, v \in U_{n, 0}$ be distinct. By $(\mathrm{r} 3)$, there is $w \in U_{n, k}$ such that $(u, w) \in \operatorname{rep}(a)$ and $(v, w) \in \operatorname{rep}(b)$, thus $(u, v) \in \operatorname{rep}(a) \mid \operatorname{rep}(b)^{-1}$ (we used the already proven (iii)). The rest is completely analogous, except that to prove (iv.5) we use (r4), and to prove (iv.6) we use (r5).

By this, we have proved Theorem 1 .

Remark 1 We note that our atom structure is obtained from its restriction to the atom structure where we omit the atoms $r_{n, k}$ and $r_{n, k}^{-}$for all $n, k \in$ $\omega, 1 \leq k \leq n$. We get our $\mathcal{S}$ by splitting $\operatorname{Id}_{n, 0} \times \operatorname{Id}_{n, k}$ (and its converse) to get the "leaves". For the method of splitting in relation algebra see [1]. 
Remark 2 We describe, briefly, another example of a pair of elementarily equivalent relational algebra atom structures with non-elementarily equivalent term algebras, due to Robin Hirsch and Ian Hodkinson. (They devised this example after seeing ours [6] and we include the example with their permission.) Let $\mathcal{H}=\langle H, P, C, I\rangle$ be the atom structure where the atoms are the elements of $H:=\omega \cup\left\{1^{\prime}, x, x^{-}\right\}$, there is one identity atom, namely $I=\left\{1^{\prime}\right\}$, all atoms are self-converse except for $x, x^{-}$which are each other's converses: $C=\left\{(a, a): a \in H \backslash\left\{x, x^{-}\right\}\right\} \cup\left\{\left(x, x^{-}\right),\left(x^{-}, x\right)\right\}$, and there is one kind of forbidden diversity triple, namely $(n, x, n+1)$ for $n \in \omega$, i.e., $P=\left\{\left(1^{\prime}, a, a\right): a \in H\right\} \cup\left\{\left(a, 1^{\prime}, a\right): a \in H\right\} \cup\left\{\left(a, b, 1^{\prime}\right)\right.$ : $(a, b) \in C\} \cup\left\{(a, b, c): a, b, c \in H \backslash\left\{1^{\prime}\right\},(a, b, c) \notin F\right\}$, where the set of the Peircean transforms of the forbidden triples is $F:=\{(n, x, n+1): n \in$ $\omega\} \cup\left\{\left(n+1, x^{-}, n\right): n \in \omega\right\} \cup\left\{\left(n+1, n, x^{-}\right): n \in \omega\right\} \cup\left\{\left(x^{-}, n, n+1\right):\right.$ $n \in \omega\} \cup\{(n, n+1, x): n \in \omega\} \cup\{(x, n+1, n): n \in \omega\}$. Let $\mathcal{H}^{\prime}$ be another atom structure which is elementarily equivalent to $\mathcal{H}$ but in which there is a non-well-founded model $\omega^{+}$instead of $\omega$. In both term algebras the elements are finite and cofinite sets of atoms. One can express that $n$ and $m$ are selfconverse diversity atoms and $m=n+1$, by using the forbidden triangles. Let $\varphi$ be the formula that says that for all self-converse non-identity atoms $n$ there is an element closed under predecessors and containing $n$ but not $n+1$. This formula is true in the first term algebra (the element is $\{0,1,2, \ldots, n\}$ ) but false in the second term algebra.

\section{References}

[1] Andréka, H., Maddux, R.D., Németi, I., Splitting in relation algebras. Proceedings of Amer. Math. Soc. 111,4 (1991) 1085-1093.

[2] Bezhanishvili, N., Varieties of two-dimensional cylindric algebras. In: Cylindric-like algebras and algebraic logic., eds: Andréka, H., Ferenczi, M., Németi, I., Bolyai Society Mathematical Studies Vol 22, Springer Berlin Heidelberg New York 2013, pp.37-59.

[3] Givant, S., Advanced topics in relation algebras. Springer International Publishing AG, 2017, xix + 605 pp.

[4] Henkin, L., Monk, J.D., Tarski, A., Cylindric Algebras Part I, NorthHolland, Amsterdam, 1971. 
[5] Hirsch, R., Hodkinson, I., Relation algebras by games. Studies in Logic and the Foundations of Mathematics, vol. 147, Elsevier Science, NorthHolland Publishing Company, Amsterdam (2002)

[6] Hirsch, R., Hodkinson, I., Letter to the authors. October 23, 2017.

[7] Jónsson, B., Tarski, A., Boolean algebras with operators. Part I: Amer. J. Math. 73 (1951), 891-939. Part II. Amer. J. Math. 74 (1952), 127-162.

[8] Maddux, R.D., Some varieties containing relation algebras. Trans. Amer. Math. Soc. 272,2 (1982), 501-526.

[9] Maddux, R.D., Relation algebras. Studies in Logic and the Foundations of Mathematics, vol. 150. Elsevier Science, North-Holland Publishing Company, Amsterdam (2006)

[10] Simon, A., Non-representable algebras of relations. PhD Dissertation, Hungarian Academy of Sciences, Budapest, 1997. iii+86pp.

[11] Venema, Y., Atom structures. In: Kracht, M., de Rijke, M., Wansing, H., Zakharyaschev, M., eds., Advances in Modal Logic 96. pp.291-305. CSLI Publications, Stanford, 1997.

Alfréd Rényi Institute of Mathematics, Hungarian Academy of Sciences Budapest, Reáltanoda st. 13-15, H-1053 Hungary

andreka.hajnal@renyi.mta.hu, nemeti.istvan@renyi.mta.hu 